\title{
La transversalidad en la formación en estudiantes de doctorado. Experiencias innovadoras en la gestión de la propia formación.
}

\author{
Carmen Corona-Sobrino ${ }^{a}$ y Gonzalez-Urango, Hannia ${ }^{b}$ \\ ${ }^{a}$ INGENIO (CSIC-UPV), Universitat de València. carcosob@ingenio.upv.es, bINGENIO (CSIC- \\ UPV), Universitat Politècnica de València. hangonur@upv.es
}

\begin{abstract}
The model for assessing the quality of science and higher education in Spain has derived a model for evaluating academic careers based on three main themes: research, teaching and management. The latter is the most difficult to strengthen during the predoctoral training stages. The innovation experience we present is about the latter and is developed within the framework of a scientific congress. The realization of this international event "by and for" researchers in predoctoral training meant the congregation of more than 50 researchers in the early stage, in areas related to Science, Technology, Innovation and Social Sciences. The organizers highlight the value of the experience mainly thanks to the strengthening of some skills required in the later stages of the academic career and that are outside the day to day of a researcher in the predoctoral category. In general, aspects such as decision making, communication, management and teamwork. Which are hardly reinforced during the training of a PhD, they were highly favored.
\end{abstract}

Keywords: competences, training, metodology, evaluation, PhD, events management, scientific congress, teaching innovation, predoctoral training, academic careers.

\footnotetext{
Resumen

El modelo de evaluación de la calidad de la ciencia y la educación superior en España ha derivado un modelo de evaluación de la carrera académica basado en tres grandes ejes temáticos: la investigación, la docencia y la gestión. Este último es el mas difícil de fortalecer durante las etapas de formación predoctoral. La experiencia de innovación que presentamos versa sobre esta ultima y se desarrolla en el marco de la realización de un congreso científico. La realización de dicho evento internacional "por y para" investigadores en formación predoctoral significó la congregación de mas de 50 investigadores en etapa de formación, en áreas relacionadas con Ciencia, Tecnología, Innovación y Ciencias Sociales. Las organizadoras destacan el valor de la experiencia principalmente gracias al fortalecimiento de algunos habilidades requeridas en las etapas posteriores de la carrera académica y
} 
La transversalidad en la formación en estudiantes de doctorado. Experiencias innovadoras en la gestión de la propia formación.

que están fuera del día a día de un investigador en la categoría predoctoral. En general, aspectos como la toma de decisiones, la comunicación, la gestión y el trabajo en equipo; que son difícilmente reforzados durante la formación del doctorado, se vieron altamente favorecidos.

Palabras clave: competencias, formación, metodología, evaluación, doctorado, gestión de eventos, congreso científico, innovación docente, formación predoctoral, carrera academica.

\section{Introducción}

La institución universitaria española ha experimentado diversos cambios en las últimas décadas: la expansión y crecimiento de la institución, el aumento de los flujos de movilidad tanto de los estudiantes como del personal, un aumento de la investigación dirigida por la academia y una creciente presión por el rendimiento de cuentas ante la sociedad (Paradeise y Thoenig 2016). Estas transformaciones han afectado a las distintas misiones de la institución, a los procesos internos de administración y gestión, y a su posicionamiento en un mercado global de la educación, entre otros.

Actualmente, la universidad se encuentra en la búsqueda y consolidación de un sistema mejor financiado, con estructuras de gobernanza modernas y ágiles, cuyo objetivo es implementar políticas que favorezcan la diferenciación, la especialización y la excelencia en el panorama internacional (Gobierno de España 2010). Asimismo, debido a la coyuntura económica y social, tanto la sociedad como el gobierno reclaman mecanismos de justificación de los gastos de los recursos que se le han asignado (Bricall 2000). Para cumplir con estas reformas, se han impulsado una serie de indicadores, incentivos y procedimientos de evaluación, con el fin de mejorar la asignación de los recursos, valorando principalmente el rendimiento (Paradeise y Thoenig 2016). Ha supuesto el afianzamiento de nuevas metodologías de evaluación que tratan de objetivar el desempeño y competir con los nuevos requisitos del sistema.

En este contexto se configura el Sistema de Evaluación de la calidad de la Investigación y la educación superior (SEI) en España que comienza a desarrollarse en la Transición Democrática a través de una serie de leyes cuya finalidad era modernizar tanto a la ciencia como a la propia institución universitaria. Los SEI son utilizados desde entonces como marcadores de la política científica, como herramientas distributivas de recursos y recompensas, como elementos estratégicos para evaluar el rendimiento de las universidades o, en el caso que aquí nos ocupa, en un nivel micro de evaluación individual del académico o investigador. Las agencias de evaluación han establecido una serie de programas con sus respectivos criterios de evaluación, a través de los cuáles se van consiguiendo una serie de acreditaciones para el acceso o desarrollo en la carrera académica. Los tres grandes ejes temáticos de la evaluación son la investigación, la docencia y la gestión. Es este último sobre el que versa la experiencia de innovación ha presentar, desarrollada en el marco de la realización de un congreso científico. 
A continuación presentamos una breve introducción al SEI español para ofrecer una visión en conjunto de la importancia de la innovación aquí planteada: la realización de un congreso científico internacional "por y para" estudiantes de doctorado.

\subsection{La evaluación de la carrera académica en España}

Los procesos de evaluación son fundamentales para el desarrollo de la carrera académica. La información que proporcionan, gracias a la estandarización de los criterios y significados de calidad, adquiere especial relevancia en la política de recursos humanos. En el caso español, la información proporcionada es utilizada para la obtención de la acreditación, que habilita para el acceso y promoción dentro de los cuerpos docentes universitarios. También se otorga, una compensación económica por el desempeño docente e investigador, denominada sexenio, si bien no es objeto de nuestro análisis.

La carrera académica en el Sistema Universitario Español (SUE) se configura como muestra la Figura 1. Este es el procedimiento estandarizado si bien existen diferentes interpretaciones a la normativa (Cañibano et al. 2017). La lógica es la siguiente: una vez se ha obtenido el título de doctor es necesario obtener el certificado de acreditación qué es obligatorio para todas las categorías profesionales. En el momento en el que el investigador cuenta con una evaluación positiva puede presentarse al concurso de acceso, obligatorio también en todos los departamentos universitarios.

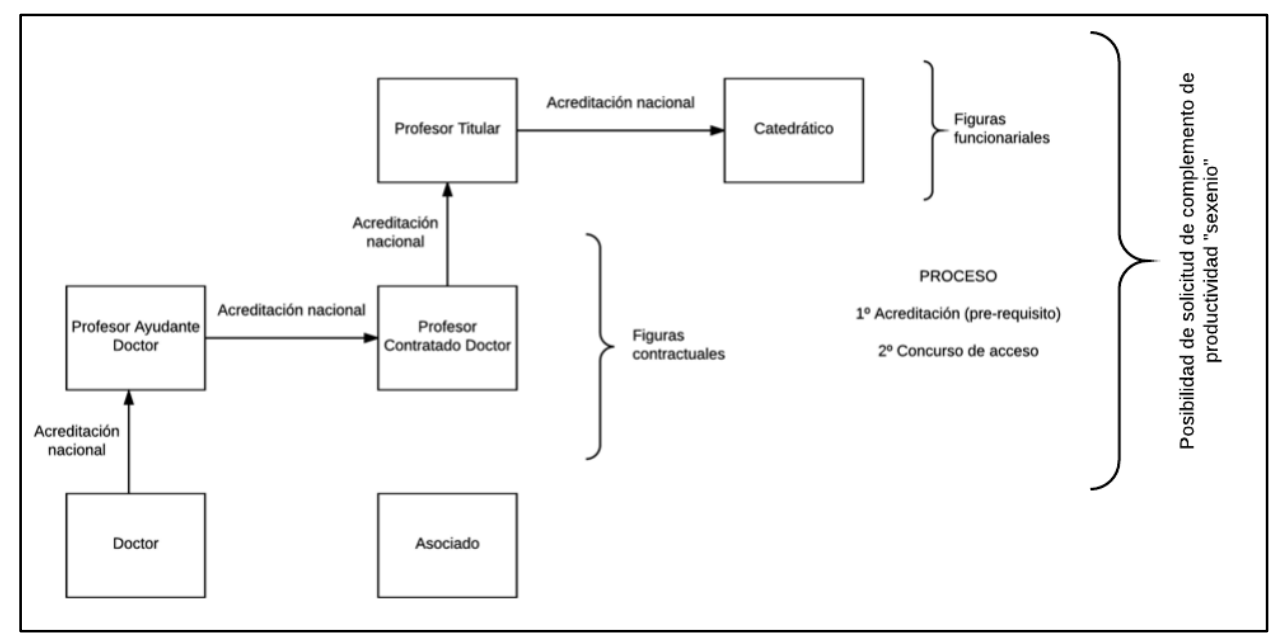

Fig. 1 Desarrollo estándar de la carrera académica según la normativa vigente (LOU 4/2007). Elaboración propia

Para obtener la acreditación nacional se tienen en cuenta los tres ámbitos de trabajo académico principales: la docencia, la investigación y la gestión. En esta comunicación nos centraremos en el apartado de la gestión, en cómo empezar a trabajarla desde un momento concreto de la carrera académica, el doctorado. La gestión puede ser realizada de distintos modos, desde la ocupación de cargos académicos como temas de administración científica, educativa y tecnológica. En esta comunicación, presentamos la gestión referida a la 
organización de un evento científico pero con una particularidad e innovación debido a que es un evento gestionado por los propios estudiantes de doctorado hacia exclusivamente otros estudiantes de doctorado.

\subsection{La organización de un evento científico}

La carrera académica presenta diversos desafíos y una variedad de trabajos a realizar en la misma (Gläser y Laudel 2015). Desde la redacción de un paper científico, pasando por la impartición de clases de máster, hasta la ocupación de un puesto en el Rectorado son todas actividades que forman parte de la vida laboral de un académico. En este sentido, la organización de eventos científicos adquiere una especial relevancia dado el carácter el quehacer investigador, basado en la dación y exposición de las propias investigaciones a la comunidad científica con el objetivo de contribuir al fin social de la ciencia fundamentado en la construcción conjunta del conocimiento.

De esta manera, los congresos científicos se convierten en un elemento indispensable para la divulgación de la propia investigación, la exposición a la crítica y al debate, y para la creación de redes sociales con otros investigadores de la especialidad. Son múltiples los congresos, simposios o reuniones científicas que existen a nivel regional, nacional e internacional, sin embargo, no suelen estar enfocados exclusivamente a estudiantes de doctorado sino que dentro de estos eventos se adecúan sesiones para la presentación de los mismos. Estas sesiones muchas veces son residuales dentro de los eventos y no cuentan con la participación adecuada de los investigadores seniors o de los propios estudiantes, además no favorece en gran medida un clima de aportación o contribución. Dada esta carencia y debido a la importancia de la formación en la participación de este tipo de eventos, se ha considerado necesario el realizar un evento propio que cree una atmósfera que estimule la participación, el diálogo y la presentación de resultados o aportaciones teóricas iniciales sin la presión o coerción social que puede generar un evento científico más senior. Esta es la filosofía a raíz de la que nacen los denominados "PhD Days".

\subsection{Presentación de los PhD Days}

Los "INGENIO PhD Days" son jornadas de encuentro de estudiantes de doctorado que organiza bienalmente el Instituto de Gestión de la Innovación y del Conocimiento INGENIO (CSIC - Universitat Politècnica de València), institución cuya actividad científica se enmarca en el campo de la Ciencia, la Tecnología y la Innovación.

El evento está dirigido a estudiantes de doctorado pertenecientes a universidades, institutos y otras organizaciones o entidades académicas, en el ámbito autonómico, nacional e internacional, que desarrollen su investigación en las áreas de Ciencia, Tecnología, Innovación y Ciencias Sociales.

Este espacio de encuentro concebido a partir de los casos exitosos de otros centros similares en Europa, como DRUID-DIME Academy Winter Conference (Aalborg University, Copenhagen Business School, University of Southern Denmark), y Science and Technology 
Policy Research Unit (SPRU- University of Sussex); ha logrado abrirse un espacio entre una nueva generación estudiantes de doctorado procedentes de diversas universidades y centros de investigación europeos que han sido parte de las tres ediciones anteriores. El primer encuentro celebrado en 2013 contó con 30 estudiantes, mientras que la última edición fueron 35 los participantes, más de la mitad de los mismos procedentes de países de la Unión Europea.

La cuarta edición que aquí se presenta, se denominó "2018 INGENIO PhD Days: Science, Technology and Innovation: new challenges and practices“ contribuyó al proceso de institucionalización del evento a lo largo del tiempo y su consolidación en las redes académicas internacionales. Se celebró en las instalaciones de la Ciudad Politécnica de la Innovación (CPI) en Valencia del 3 al 5 de Mayo del 2018 y contó con mas de 60 participantes entre presentadores y asistentes.

\subsection{Finalidad del evento}

El evento va dirigido a cuatro públicos interrelacionados:

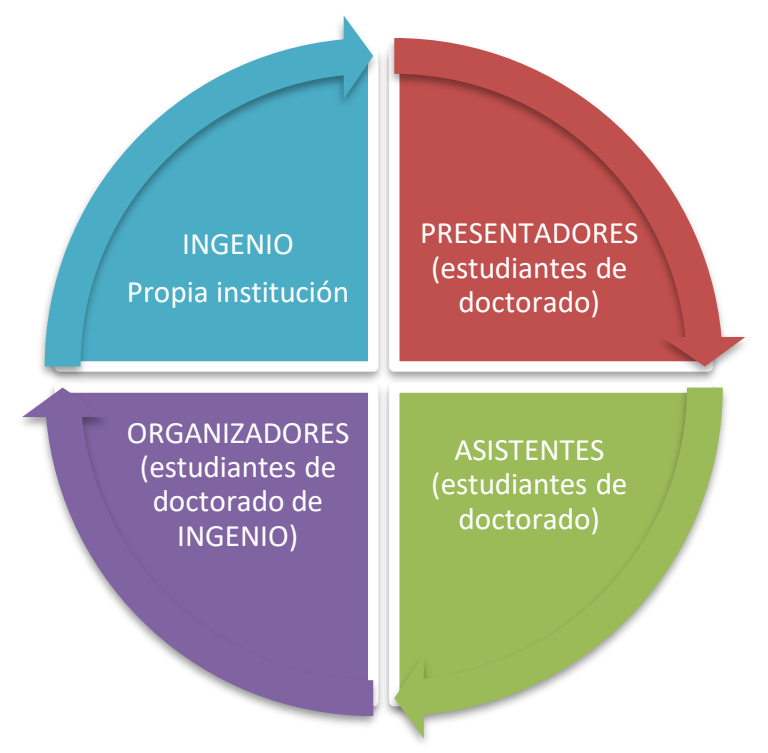

Fig. 2 Públicos interrelacionados. Elaboración propia

El objetivo general de los "PhD Days", es apoyar la formación de la próxima generación de investigadores en las áreas de Ciencia, Tecnología, Innovación y Ciencias Sociales aplicados a estudios de innovación y gestión del conocimiento; enmarcado en el Plan Estratégico del Instituto INGENIO (CSIC-UPV).

En concreto, este evento tuvo como objetivos específicos los siguientes: 
La transversalidad en la formación en estudiantes de doctorado. Experiencias innovadoras en la gestión de la propia formación.

- Mejorar las perspectivas de carrera de los jóvenes investigadores, tanto en el sector público como privado, haciendo más atractiva la carrera investigadora mediante mecanismos de networking trans - nacional.

- Fortalecer las relaciones entre expertos y estudiantes de doctorado en las áreas relacionadas.

- Propiciar la reflexión en torno al compromiso y los nuevos desafíos de la ciencia, la innovación y la universidad en el cambio social.

- Fortalecer la participación y la influencia en el ámbito local y regional reforzando vínculos con los beneficiadores potenciales de la investigación en un entorno próximo.

- Establecer un diálogo entre diferentes comunidades y corrientes científicas, que permita conocer sus perspectivas y aportes para responder a los nuevos desafíos de la sociedad.

- Explotar el carácter multidisciplinar del evento con el fin de abrir nuevos horizontes y nuevas oportunidades para los participantes y para el centro.

- Potenciar la imagen del Instituto y contribuir al fortalecimiento de la relevancia social y científica de las áreas de investigación claves.

- Propiciar la creación y consolidación de redes con otras instituciones con denotada reputación internacional.

- Aumentar la visibilidad del Instituto con el fin de mejorar la atracción de futuros talentos investigadores.

\section{Objetivos de la comunicación}

Esta comunicación tiene los siguientes objetivos:

- Presentación de una experiencia exitosa para la formación de los estudiantes de doctorado.

- Valoración de la experiencia en la organización, gestión y difusión del evento como parte de la formación integral de la etapa doctoral.

\section{Desarrollo de la innovación}

La innovación de este evento como ya se ha indicado reside en la realización y gestión del evento por los propios estudiantes de doctorado de INGENIO. Esto ha incluído todas las fases de la preparación, elaboración y gestión del evento, así como la solicitud y consecución de financiación para la realización del mismo. Se exponen a continuación las distintas fases de desarrollo de la innovación.

\subsection{Fase de organización}

El Comité Organizador de los PhD Days INGENIO 2018 estuvo conformado exclusivamente por estudiantes de doctorado de INGENIO (CSIC-UPV): 
- Carmen Corona Sobrino (CSIC-UPV)

- Elena Denia Navarro (CSIC-UPV)

- Hannia Gonzalez Urango (UPV)

- Victoria Pellicer Sifres (CSIC-UPV)

La fase inicial para la realización del evento tuvo las siguientes fases clave:

- Redacción de la propuesta científica. El lanzamiento del "Call for abstracts" requirió de un proceso intelectual para la selección del tema central del evento y la unión de los subtemas de manera que quede un programa científico sólido y con una consistencia y coherencia temática. Además, en este momento se estableció un Comité Científico, conformado por investigadores seniors,encargado de la revisión de las propuestas que se recibían evaluándolas y proporcionando un feedback adecuado.

- Solicitud de la financiación. Una vez se tiene un programa científico sólido se procede a la solicitud de la financiación y la elaboración del presupuesto correspondiente. En este caso, además de la parte autofinanciada del evento las organizadoras solicitaron financiación a EuSPRI (red de investigación europea) y a la Generalitat Valenciana. Ambas fueron aceptadas, sin embargo, dado el carácter de exclusividad de la financiación el evento se llevo a cabo con la dotación presupuestaria de la red de EuSPRI.

- Difusión del evento (ver también apartado 2.3). En este momento se contó con el apoyo institucional del centro además de las redes de contactos de los investigadores.

- Recepción de los abstracts, valoración por parte del Comité Científico y selección de propuestas. Los y las estudiantes que desearon participar enviaron los resúmenes de sus propuestas de investigación, las cuales fueron evaluadas por el comité científico teniendo en cuenta su adecuación a los temas propuestos.

- Organización de aspectos académicos del evento: selección de paneles y elección de los ponentes y de los talles de formación.

- Organización de aspectos formales del evento: catering, programa social, merchandising del congreso, realización de material audiovidual...

\subsection{Estructura y alcance del Congreso}

El encuentro estuvo estructurado en cuatro tipos de actividades: ponencias magistrales, talleres de formación, paneles de debate con los doctorandos, y una reunión con editores y editoras. Las ponencias magistrales estuvieron a cargo de dos reconocidos científicos a nivel internacional, que aportaron dos intervenciones de alta calidad científica acordes con la temática central. Los talleres de formación fueron dos espacios que trataron sobre temáticas prácticas de amplio interés para investigadores/as en proceso de formación, impartidos por profesionales con experiencia en los temas a tratar.

Por su parte, los paneles de debate, fueron espacios donde los y las estudiantes asistentes expusieron sus trabajos y obtuvieron feed-back tanto de los asistentes a la sesión como del 
investigador o investigadora senior que dirigió la sesión (uno o una por panel). Los trabajos que se expusieron se seleccionaron con anterioridad al inicio del evento a través del ya mencionado call for abstracts (llamado abierto) (para más detalle de los temas visitar la web http://www.ingenio.upv.es/teams/phd-students/ingenio-phd-days-2018). Y por último, como una de las principales novedades de esta nueva edición, se realizó un encuentro de estudiantes con editores y editoras de revistas científicas en áreas relacionadas; un espacio donde los participantes pudieron intercambiar opiniones y experiencias, y los y las estudiantes pudieron hacer consultas, aclarar inquietudes y recibir consejos relacionados con la publicación de trabajos científicos.

\subsection{Difusión del evento}

Para la difusión del evento, se creó una plataforma web que contuvo toda la información referida al encuentro: programa, actividades, talleres, datos del comité científico y organizador etc. También se contó con información práctica relativa a la Universitat Politècnica de València y a la ciudad de Valencia mediante la herramienta Politube. El sistema de registro multiusuario permitirá que todos aquellos posibles participantes puedan subir sus propuestas, y una vez aceptadas, puedan acceder a información más detallada.

Se creó una página web exclusiva para el evento http://www.ingenio.upv.es/teams/phdstudents/ingenio-phd-days-2018 y como lanzamiento del mismo se realizó un vídeo donde los estudiantes de doctorado contaban sus experiencias respecto a ediciones anteriores y la filosofía del evento https://www.youtube.com/watch?v=WR3-Px7nT_c\&t=116s

Además se utilizaron las redes de difusión propias del instituto de investigación INGENIO (CSIC-UPV):

- TWITTER: @Ingenio_CsicUpv

- FACEBOOK: https://www.facebook.com/INGENIO.CSIC.UPV/

- Newsletter semanal y cuatrimestral de INGENIO

- Newsletter semanal y trimestral de Delegación CSIC Comunidad Valenciana

○ Página web INGENIO: http://www.ingenio.upv.es/es

○ Canal Youtube: Ingenio Csic Upv

\section{Resultados}

\subsection{Aspectos académicos del evento}

En líneas generales tanto la organización como la celebración del evento han sido consideradas como ampliamente satisfactoría tanto por los organizadores como por los participantes. En torno a 50 abstract fueron recibios de un amplio y variado número de instituciones y países. Después de las evaluaciones del Comité Científico, 43 contribuciones fueron las seleccionadas y agrupadas en 11 paneles diferentes. La valoración de la calidad de las contribuciones aceptadas fue notablemente elevada, los participantes obtuvieron un 
feedbak útil y comentarios constructivos antes de la llegada al congreso y después durante sus presentaciones.

Los participantes tuvieron la oportunidad de establecer estimulantes e intelectuales conversaciones entre ellos y con los seniors del Instituto INGENIO durante los tres días de duración del evento. Los temas principales fueron relacionados con los temas de investigación presentados así como el desarrollo de sus actividades académicas y estrategias futuras.

\subsection{Actividades sociales}

Además del aspecto académico, durante el evento hubo tiempo para la realización de actividades de carácter social que afianzaron las redes de contacto entre los estudiantes y los seniors. Como viene siendo habitual en este tipo de eventos se realizó un "coctail de bienvenida" y una "cena social" en los cuales se intercambiaron conversaciones y se potenció la red de contactos. Además se realizó un concierto al finalizar el evento en la terraza de la CPI con el fin de amenizar la última comida del congreso. Estas actividades fueron muy bien valoradas y apreciadas por los participantes según ha quedado registrado en la encuesta de satisfacción, ya que consideran que hubo mucho interés por parte de los organizadores en favorecer este tipo de encuentros más informales para establecer conversaciones.

\subsection{Encuestas de evaluación de los participantes}

Se diseñó y distribuyó a todos los participantes una encuesta con el fin de recoger todas sus opiniones y comentarios sobre la organización y el contenido del evento. El formulario tenía 10 partes diferenciadas para evaluar distintos aspectos en una escala de 1 a 4 ( $1=$ very bad/not at all; $4=$ very good/a lot $)^{1}$. Los aspectos que se evaluaron fueron los siguientes: la calidad científica del evento, el feedback recibido, el networking, los Keynote speech \& Workshops, y la organización y el programa social.

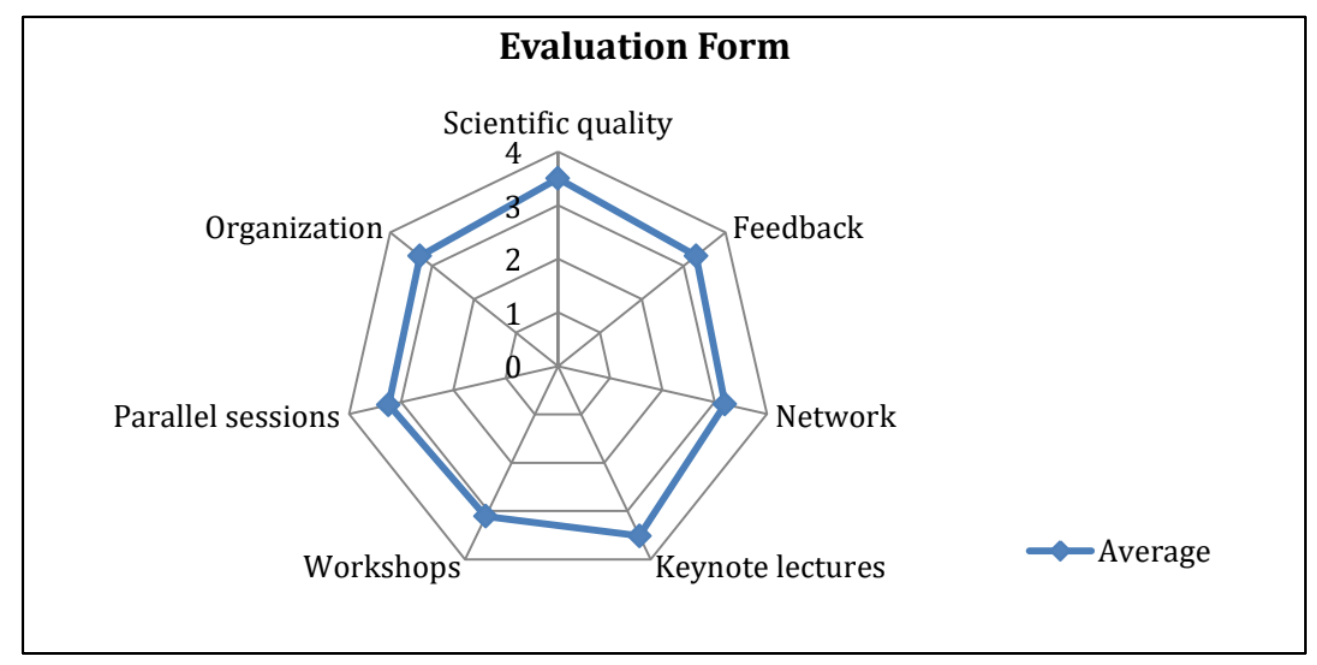

Fig. 3 Resultados de la evaluación general

\footnotetext{
${ }^{1}$ Dado el carácter internacional del evento toda la documentación del mismo está en inglés
} 
Se recogieron también comentarios de tipo cualitativo:

- "Thanks for your effort in organizing that event, I really enjoyed it".

- "Overall a very good event, better than others in the area, above average, good job".

- "It was very well organized. Thanks a lot! A great event in terms of getting feedback, learning useful and interesting things and social activities".

\section{Conclusiones}

Las conclusiones de la comunicación que aquí se presenta vienen dadas por parte de dos de las organizadoras del Congreso "PhD Days 2018", que son a su vez las autoras de dicha presentación. La organización de este tipo de eventos en este momento de comienzo de la carrera académica ha supuesto un reto, mayor aún cuando es única y exclusivamente realizado por parte de estudiantes de doctorado. Esta experiencia es valorada como muy satisfactoria y útil para el desarrollo futuro de la carrera académica y para el establecimiento de redes de contactos.

La gestión es un aspecto que queda siempre más alejado de la formación del estudiante de doctorado pero, como hemos visto en la introducción teórica, es una parte fundamental para el progreso dentro de la carrera académica. Por ello, consideramos que la oportunidad y el apoyo ofrecido por el centro de investigación INGENIO, como parte de su política de formación integral, para el desarrollo del evento es fundamental y está en líneacon las directrices que vienen marcando las Agencias de evaluación estatales y autonómicas.

Finalmente nos gustaría destacar algunos de los principales aportes que la organización de este evento ha representado en la formación como estudiantes de doctorado en diferentes ambitos. Por una parte, ha significado un reto y un esfuerzo enfrentarse a la organización de un evento de estas características, que implica la toma de decisiones importantes que un evento requiere. Otro de los ambitos a destacar, es el trabajo en equipo, como estudiantes de doctorado dificilmente nos vemos en la necesidad de realizar trabajos en conjunto con otros compañeros en la misma etapa de formación. Además, la comunicación constante con otros estudiantes e investigadores seniors, supuso un aporte importante en el fortalecimiento de las habilidades de comunicación. Y por ultimo, el aprendizaje en la solicitud de financiación y todos los aspectos indirectos relacionados con la gestión y administración generados;consideramos fundamental e interesante el acercamiento a aspectos más administrativos, como la realización de un presupuesto. En general los ambitos anteriores están fuera del día a día de un investigador en la categoría predoctoral, sin embargo, son necesarios en las etapas posteriores de la carrera académica, en donde esperamos poder desarrollarnos desde diferentes roles en la investigación, la docencia y la gestión

\section{Referencias}

Bricall, Josep. 2000. Informe Universidad 2000. Barcelona. Recuperado (http://www.oei.es/oeivirt/bricall.htm). 
Cañibano, Carolina, Carmen Corona, Jordi Molas-Gallart, y Immaculada Vilardell. 2017. «Dinámicas organizativas en los departamentos universitarios españoles: diversidad estratégica y rigideces institucionales». Ekonomiaz 92:274-300.

Gläser, Jochen y Grit Laudel. 2015. «The Three Careers of an Academic». Discussion paper. Berlin: TU Berlin, Center for Technology and Society (35).

Gobierno de España. 2010. Estrategia Universidad 2015. Universidades para el progreso, el bienestar y la competitividad. España.

Paradeise, Catherine y Jean-Claude Thoenig. 2016. In search of academic quality. Palgrave Macmillan UK. Recuperado (https://www.scopus.com/inward/record.uri?eid=2-s2.084981513611\&partnerID=40\&md5=f93fe6b1da800038af94d1716e0cb437). 